\title{
Research on Suppression Method of Warhead Infrared Image Background Based on Small Area Filtering
}

\author{
Yehui Li, Shaohua Sun \\ Branch 30, Unit 91049, Qingdao, China \\ Email: happysunshao@163.com
}

How to cite this paper: Li, Y.H. and Sun, S.H. (2018) Research on Suppression Method of Warhead Infrared Image Background Based on Small Area Filtering. Journal of Computer and Communications, 6 , 155-161.

https://doi.org/10.4236/jcc.2018.611015

Received: November 5, 2018

Accepted: November 12, 2018

Published: November 19, 2018

\begin{abstract}
In order to reduce the computational complexity of searching in massive information in detecting of warhead targets, background removal is usually the first step of target detection algorithm in sequential frame images. In this paper, an adaptive multi-exposure time preserving star edge small area filtering background removal algorithm is proposed, which can suppress the background and preserve the target and star edges. This algorithm not only ensures the accuracy of centroid and orbit determination, but also reduces false alarm and improves tracking accuracy.
\end{abstract}

\section{Keywords}

Warhead, Infrared Image, Suppression Method

\section{Introduction}

In order to complete the warhead targets detection, it is necessary to pre-process the image before implementing the specific target detection algorithm. The pre-processing is the premise of target detection. The purpose is to suppress the noise in the image and improve the signal-to-noise ratio of the target [1]. In view of various complex background conditions, there are many kinds of image preprocessing methods for dim and small targets, and these methods have different characteristics. It is these differences that lead to differences in the applicability of research objects [2]. None of the existing methods can show the best preprocessing effect for all complex background. In application, different pretreatment methods should be chosen according to specific background conditions. Infrared image preprocessing mainly focuses on inducing of image noises and image enhancement. 
Usually, a threshold is set in background removal. If the pixel is smaller than the threshold, it is considered as the background. If the threshold is larger than the threshold, it is considered as the target. Threshold setting methods include optimal threshold and local optimal threshold. Another method uses the background modeling method to estimate the mean and standard deviation of the background, set a global threshold, and use the statistics of the neighborhood of the point to judge whether the background is or not. It is essentially a transformation of the background and the target probability density function, making it more conducive to highlighting the target and suppressing noise. In this paper, a new algorithm based on small area filtering is proposed, which uses global threshold [3]. To some extent, the algorithm is not sensitive to threshold. Under the same threshold, it can reduce $70 \%-80 \%$ noise points compared with direct threshold segmentation.

\section{Imaging Model for Space Targets}

The exposure time of the experimental image data in this paper is $300 \mathrm{~ms}, 700$ $\mathrm{ms}, 1100 \mathrm{~ms}$ and $1600 \mathrm{~ms}$ respectively, and the corresponding frames are 352, 217, 281 and 302. The image size is $1024{ }^{\star} 1024$, and the pixel gray level is 16 bits. Figure 1 gives a typical image of exposure time of 4 files (only suitable gray level segments are displayed for easy observation) and its gray level histogram [4]. From the statistical results of a single frame histogram, the histogram is Gaussian distribution, and the probability density function is shown in Formula (1).

$$
p(s(x, y))=\frac{1}{\sqrt{2 \pi} \sigma} e^{-(s(x, y)-u)^{2} / \sigma^{2}}
$$

$s(x, y)$ represents the gray value of points whose coordinates is $(x, y), u$ and $\sigma$ represents the mean value and standard deviation of the background respectively.

According to a large number of statistics, most of the images belong to the background, where stars and targets only account for a small part. The ratio of stars and target pixels to total primes does not exceed 3\%.

The signal of noise ratio (SNR) can be calculated according to the formula of SNR (2).

$$
S N R=20 \log _{10} \frac{T-B}{\sigma}
$$

$T$ denotes the mean value of targets, including background. $B$ denotes mean value of the neighborhood background. $\sigma$ represents the standard deviation of neighborhood background, where the neighborhood takes the target centroid region to expand 10 pixels, excluding the target itself.

\section{Estimation of the Mean Value and Variance of Background with Least Squares Fitting Method}

From the statistical results of the image, it is concluded that the gray distribution of single pixel is very wide $(150-50,000)$, the background is in accordance with 
Gaussian distribution, and some stars have very high gray level. The procedure of least squares fitting for mean and variance of background clutter is shown in

\section{Figure 1.}

Based on the above methods, the statistical distributions of mean and standard deviation of background noise in 1152 frames of $300 \mathrm{~ms}, 700 \mathrm{~ms}, 1100 \mathrm{~ms}$ and $1600 \mathrm{~ms}$ are estimated as shown in Table 1.

\section{Removal of Small Area Filter Background}

When the signal-to-noise ratio of the target is low, the gray value of the background is partially interlaced, and cannot be separated by a global threshold. If
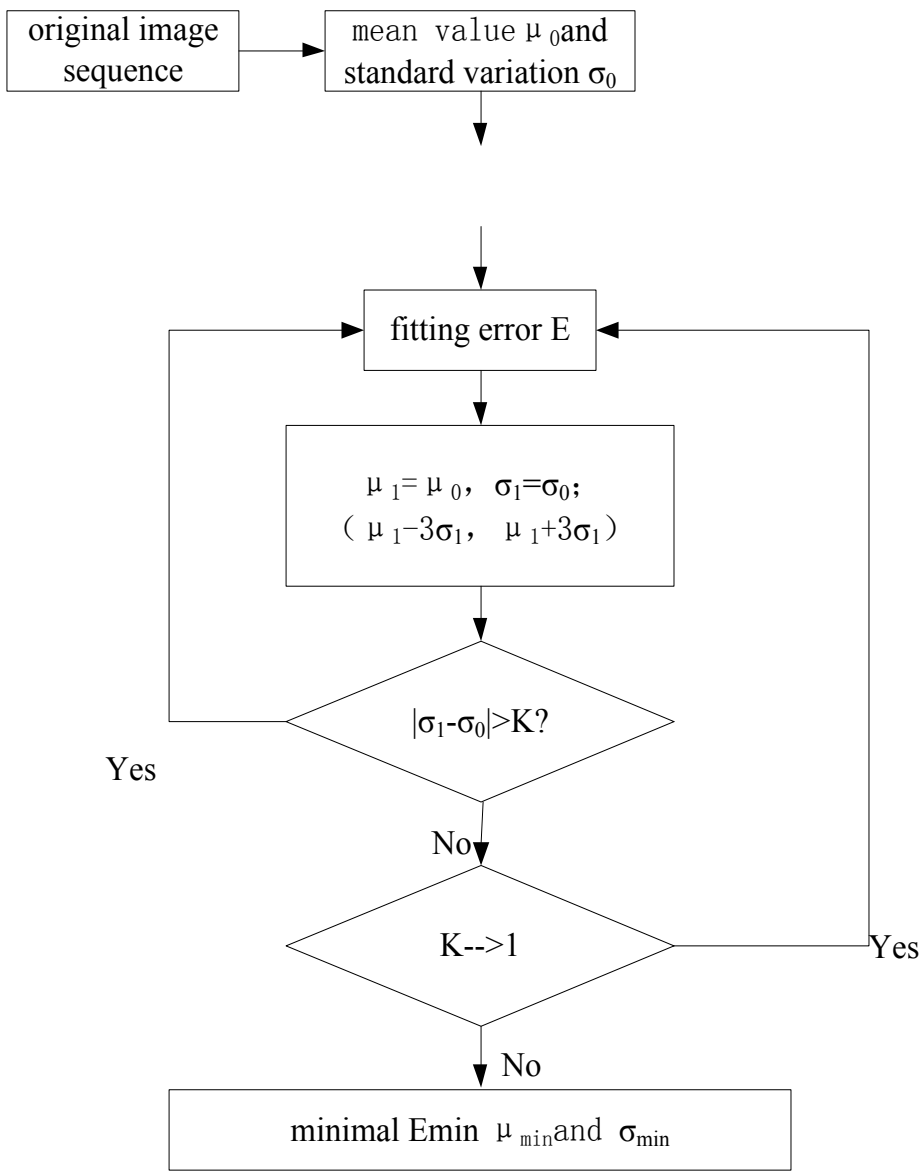

Figure 1. Flow chart of least squares estimation for background clutter parameters.

Table 1. Statistics of mean and standard deviation of background noise.

\begin{tabular}{ccccc}
\hline $\begin{array}{c}\text { exposure } \\
\text { time (ms) }\end{array}$ & frames & $\begin{array}{c}\text { maximum background } \\
\text { mean value }\end{array}$ & $\begin{array}{c}\text { minimum } \\
\text { background mean value }\end{array}$ & $\begin{array}{c}\text { maximum background } \\
\text { standard variation }\end{array}$ \\
\hline 300 & 352 & 187.132 & 175.943 & 10.182 \\
700 & 217 & 228.115 & 237.360 & 10.716 \\
1100 & 281 & 269.625 & 259.822 & 14.332 \\
1600 & 302 & 382.4960 & 357.406 & 17.486 \\
\hline
\end{tabular}


the threshold is too small, it will leave a lot of noise and too large image edge; if the threshold is too large, it will filter out the small and weak targets, and the image edge is not completely maintained, thus affecting the centroid positioning accuracy [5]. If we combine other filtering methods on the basis of direct threshold segmentation, such as filtering out the outliers whose connected area is less than a certain threshold, it is not appropriate. Although most isolated noise points are filtered out, it is difficult to ensure that small targets smaller than this threshold are not filtered out.

If we consider other filtering method such as median filtering, mean filtering, low-pass filtering, wavelet noise reducing for processing, the effect is not ideal.

Then the usual approach is to select a fixed threshold, by selecting an optimal threshold, so that the probability of such false segmentation is minimized. Assuming that an image contains only two main gray value regions (target and background), its histogram can be regarded as an approximation of the gray value probability density function $p(z)$. This probability density function is actually the sum of the 2 unimodal density functions of the target and the background. If the form of the probability density function is known, it is possible to select an optimal threshold to divide the image into two types of regions and minimize the error.

There are 1 images mixed with additive Gauss noise. Its mixed probability density equation can be expressed as:

$$
\begin{aligned}
p(z) & =P_{1} p_{1}(z)+P_{2} p_{2}(z) \\
& =\frac{P_{1}}{\sqrt{2 \pi} \sigma_{1}} \exp \left[-\frac{\left(z-\mu_{1}\right)^{2}}{\sigma_{1}^{2}}\right]+\frac{P_{2}}{\sqrt{2 \pi} \sigma_{2}} \exp \left[-\frac{\left(z-\mu_{2}\right)^{2}}{\sigma_{2}{ }^{2}}\right]
\end{aligned}
$$

Among them, $\mu_{1}$ and $\mu_{2}$ denote the average gray value of the background and the target area. $\sigma_{1}$ and $\sigma_{2}$ denote the mean square deviation of the mean value. $P_{1}$ and $P_{2}$ denote the prior probability of the gray value of the background and the target area respectively. According to the definition of probability $P_{1}+P_{2}=1$, there are five unknown parameters in the mixed probability density. If these parameters can be obtained, the mixed probability density can be determined.

Figure 2(b), Figure 2(c) illustrates that, under the same threshold, the number of false targets in the small region filter is 0 , and $T=\mu+2 \sigma$ the direct threshold segmentation results in a large number of false targets; Figure 2(b), Figure 2(d) comparison shows that even if the segmentation threshold is one more standard deviation, there are still four false targets in the graph Figure 2(d), and the edges of the star image cannot be well preserved.

Figure 3 shows the trajectory of maximum projection after small-area background filtering of multi-frame images, where the sequence contains dim and small targets with SNR which is close to 4 .

As far as detection sensitivity is concerned, compared with MTI algorithm of US space-based visible camera, the latter cannot extract the sequence of dim and small targets shown in Figure $\mathbf{3}$ as a candidate trajectory. 


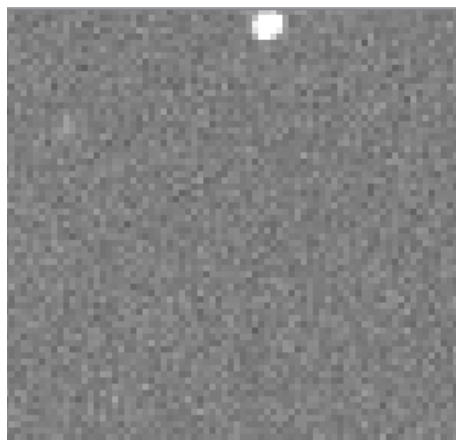

(a)

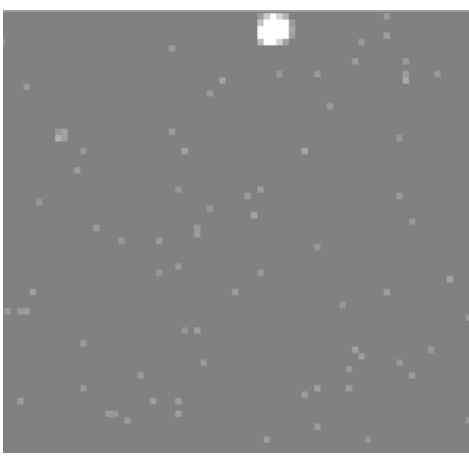

(c)

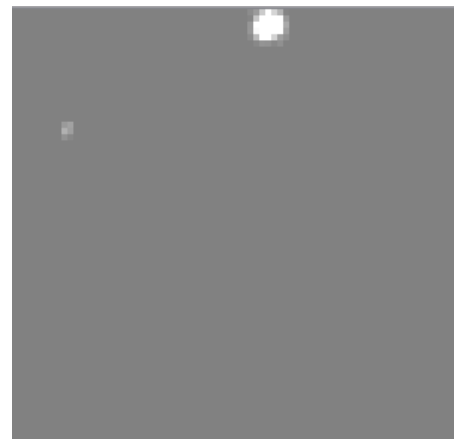

(b)

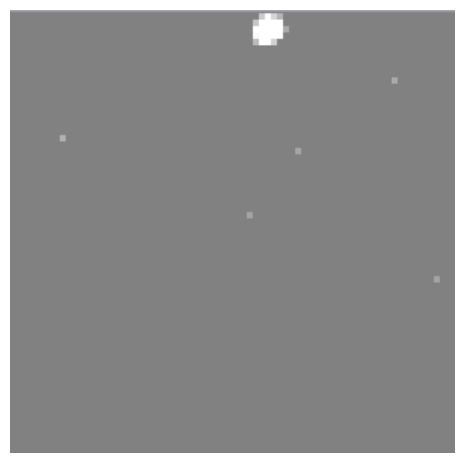

(d)

Figure 2. Comparison of three background removal techniques. (a) Original; (b) $T=\mu+2 \sigma ;(\mathrm{c}) \quad T=\mu+2 \sigma ;(\mathrm{d}) \quad T=\mu+3 \sigma$.
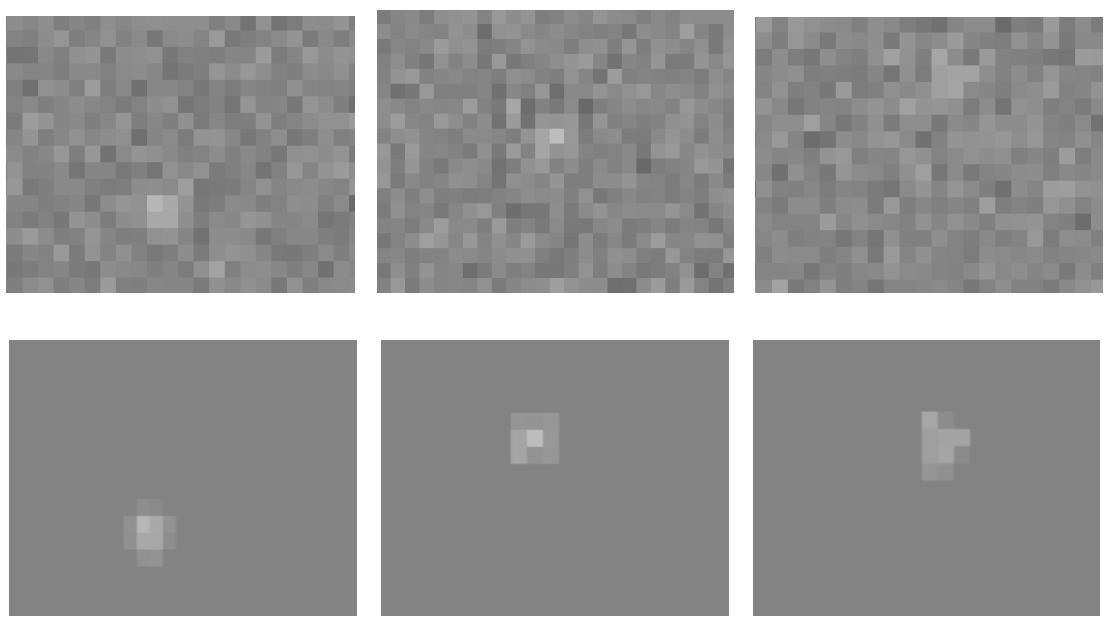

Figure 3. Segmentation results of dim and small target in three consecutive frames.

\section{Algorithm Performance}

\subsection{Sensitivity}

The algorithm sensitivity is measured by adding a simulated target with constant SNR in the image and using Monte Carlo simulation to calculate the segmentation probability of the target when the threshold $T=\mu+2 \sigma$ is fixed.

When the different scaling factors are set, the attenuation is shown in Figure 4. 


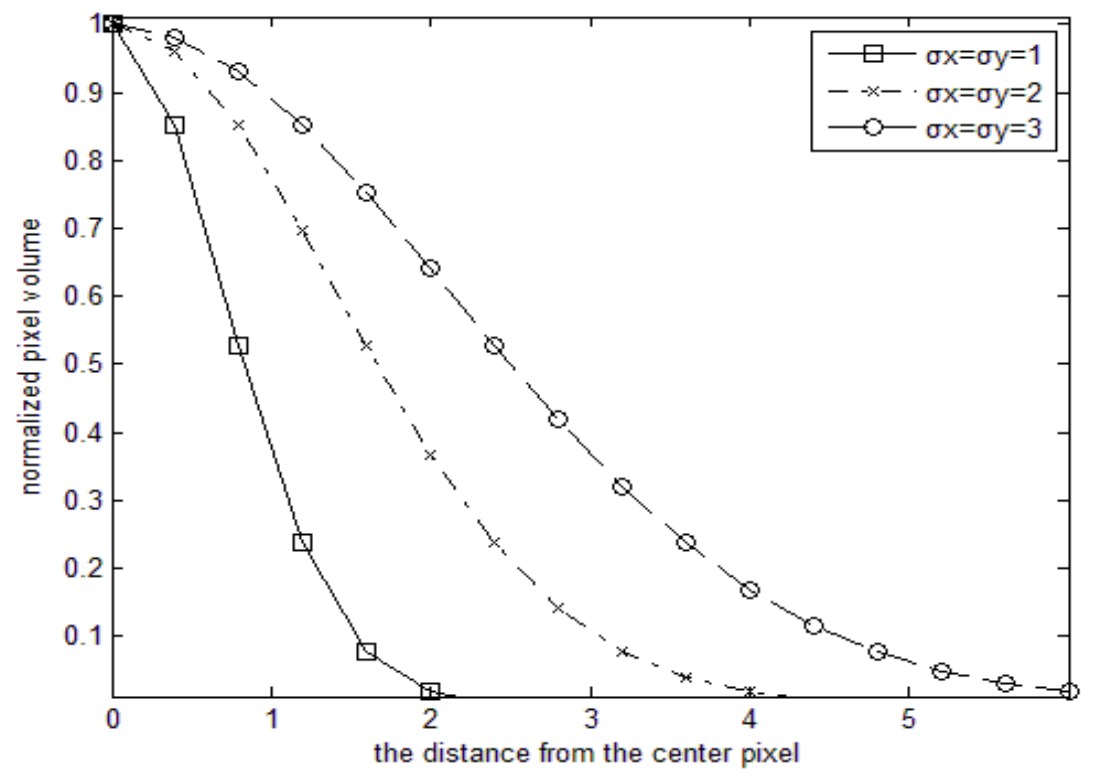

Figure 4. The pixel value of the varies with the distance from the center point.

\subsection{Time Consuming}

Headings, or heads, are organizational devices that guide the reader through your paper. There are two types: component heads and text heads.

Component heads identify the different components of your paper and are not topically subordinate to each other. Examples include Acknowledgements and References and, for these, the correct style to use is "Heading 5". Use "figure caption" for your Figure captions, and "table head" for your table title. Run-in heads, such as "Abstract", will require you to apply a style (in this case, non-italic) in addition to the style provided by the drop down menu to differentiate the head from the text. In this paper, four groups of 1152 frames are used to verify the time-consuming algorithm. The computational complexity of the algorithm is mainly on Gaussian least square fitting to estimate the mean and variance of the background. The complexity of the algorithm is mainly time-consuming statistics. Table 2 is the algorithm time consuming statistical value. The algorithm is developed under VS2005, the processor's main frequency is $1.8 \mathrm{G}$, and the memory is $2 \mathrm{G}$. Statistical results show that the algorithm has good real-time performance and meets engineering requirements.

From the statistical results, we can see that $300 \mathrm{~ms}, 700 \mathrm{~ms}, 1100 \mathrm{~ms}$ and 1600 $\mathrm{ms}$ have great differences, the main reason is that the least square fitting iteration times are different.

\section{Conclusion}

Firstly, the background noise of a single frame is statistically analyzed, and the Gaussian distribution is obtained. Then, the mean and standard deviation of the background are estimated by the least square fitting method, and the algorithm steps of the small area filtering background removal are discussed, and the ra- 
tionality of the algorithm is discussed from the point of view of the optimal threshold segmentation and likelihood ratio. After analyzing the performance of the algorithm, Monte Carlo simulation is used to verify the segmentation limit of the dim and small targets that the algorithm can reach, and the time-consuming statistical algorithm is used to process the real images. The results show that the proposed algorithm is insensitive to the global thresholding. Compared with the direct thresholding method, the proposed algorithm can reduce $70 \%-80 \%$ of the noise points when the thresholds are the same.

\section{Conflicts of Interest}

The authors declare no conflicts of interest regarding the publication of this paper.

\section{References}

[1] Chen, X.P. (2006) Russia Strengthens the Collection and Research of Characteristic Data. Target and Environmental Characteristic Management and Technical Research Reference, 07.

[2] Ping, et al. (2005) Study on Target Surface Temperature and Infrared Penetration. Aerospace Electronic Warfare, 21, 5-16.

[3] Li, K.L., Liu, Y.X. and Li, X. (2009) Target Recognition Simulation Research. Modern Radar, 28, 12-14.

[4] Liu, T., Jiang, W.D., Li, X. and Lu, H.Z. (2009) Simulation and Calculation of Target Dynamic Infrared Radiation Characteristics. Infrared and Laser Engineering, 37, 955-958.

[5] Xuan, Y.M. and Han, Y.G. (2002) Research and Application of Infrared Radiation Characteristics of Target and Background. Infrared technology, 24, 16-19. 